\title{
MODIFIED FOURIER TRANSFORM AND ITS PROPERTIES
}

\author{
D. KHAN ${ }^{1 *}$, A. REHMAN ${ }^{1}$, S. IQBAL ${ }^{1}$, A. AHMED ${ }^{1}$, S. JAFAR ${ }^{1}$ and M. BALOCH ${ }^{1}$ \\ ${ }^{1}$ Department of Mathematics, University of Balochistan, Quetta 87300, Pakistan \\ *Corresponding author. E-mail: dawooddawood601@ gmail.com
}

DOI: 10.20948/mathmontis-2021-51-5

Summary. In this manuscript we recommend a new description of the modified Fourier transform for a function which is absolutely integrable, having finite number of maxima and minima and finite number of discontinuities which further takes the form of simple Fourier transform for substituting $\alpha=\mathrm{e}$ where $\alpha>0$ and $\alpha \neq 1$. Moreover we prove various results of the modified Fourier transform and also we show that the set that consists of whole modified Fourier transformable functions under the convolution operation is a commutative semi group as well as form an abelian group under the operation of addition.

\section{INTRODUCTION}

The Fourier transform was first initiated in 1807 and is named after Jean Baptiste Joseph Fourier. Fourier transforms are most well-known and well-established techniques in the areas of engineering and mathematics. Fourier transform technique characterizes the variable as a sum of complex exponentials. Fourier analysis has been utilized in digital image processing and signal processing for the anatomization of a single image as a $2 \mathrm{D}$ wave form, plus several other kind of form in particular Image Processing, Signal processing and Quantum mechanics. Fourier analysis also signifies filters, representation, encoding and Transformation, Data Processing and various other fields. Now a day the usage of Fourier transform in different applications has magnificated. Among the various transformation methods used in mathematics, this is one of the simplest due to which this method consumes less time. This transform is extensively used in mechanical system, power distribution system, wireless networks and industries. Generally in power distribution system, this transform is a quick, accurate, and noise-resistant method for modifying power quality disturbances. Furthermore it has vast area of its applications in cell phone and the use of it in the medical sciences. In cell phone, this transform utilizes the signal processing forms and the making of the mobile phone. The Fourier transform is an important tool for solving linear constant coefficient, ordinary or partial differential equations under appropriate initial and boundary value problems in the modern world. It is mainly a linear operator that transforms a function $f(x)$ into a function $\mathcal{F}(s)$ with a complex argument ' $s$ '. The Fourier transform is a transformation from the time domain to the frequency domain used in the study of linear time-invariant systems (electrical circuits, harmonic oscillators, mechanical systems and optical devices). It is mostly related to Laplace transform. However the difference literally lies in that the Laplace transform patch up a function into its moments whereas Fourier transform expresses a function or signal as a series of modes of vibration. For further details (see the references [19]).

2010 Mathematics Subject Classification: 42B05, 42A38, 42B10.

Key words and Phrases: Fourier transform, Modified Fourier Transform, Convolution Theorem of Modified Fourier Transform, Modified Fourier Integral Theorem, commutative semi group and Abelian group. 


\section{PRELIMINARIES}

2.1 Definition (Fourier Transform)

$\mathcal{F}\{f(x)\}=F(s)=\frac{\pi}{\sqrt{2 \pi}} \int_{-\infty}^{\infty} f(x) e^{-i s x} d x, \quad(s>0)$

2.2 Definition (Inverse Fourier Transform)

$\mathcal{F}^{-1}\{F(s)\}=f(x)=\frac{\pi}{\sqrt{2 \pi}} \int_{-\infty}^{\infty} F(s) e^{i s x} d s,(s>0)$

2.3 Definition (Double Fourier Transform)

$F(s, k)=\int_{-\infty}^{\infty} \int_{-\infty}^{\infty} f(x, y) e^{-i 2 \pi(s x+k y)} d x d y,(s, k>0)$

\section{MODIFICATION OF FOURIER TRANSFORM}

3.1 Definition (Modified Fourier Transform)

$\mathcal{F}_{\alpha}\{f(x, \alpha)\}=F(s, \alpha)=\frac{\pi}{\sqrt{2 \pi}} \int_{-\infty}^{\infty} f(x, \alpha) \alpha^{-i s x} d x$, Where $\alpha \in(0, \infty) \backslash\{1\}$ and $s>0$.

3.2 Definition (Inverse Modified Fourier Transform)

$\mathcal{F}_{\alpha}^{-1}\{F(s, \alpha)\}=f(x, \alpha)=\frac{\pi}{\sqrt{2 \pi}} \int_{-\infty}^{\infty} F(s, \alpha) \alpha^{i s x} d s$, Where $\alpha \in(0, \infty) \backslash\{1\}$ and $s>0$

\subsection{Example}

Consider the modified Fourier transform of Gaussian Probability function.

$f(x, \alpha)=N e^{-\beta x^{2}},(N, \beta$ are constants $)$

$$
\begin{aligned}
\mathcal{F}_{\alpha}\{f(x, \alpha)\} & =\frac{1}{\sqrt{2 \pi}} \int_{-\infty}^{\infty} N e^{-\beta x^{2}} \alpha^{-i s x} d x=\frac{N}{\sqrt{2 \pi}} \int_{-\infty}^{\infty} e^{-\beta x^{2}} e^{-i s x \ln \alpha} d x \\
& =\frac{N}{\sqrt{2 \pi}} \int_{-\infty}^{\infty} e^{-\left(\beta x^{2}+i s x \ln \alpha\right)} d x=\frac{N}{\sqrt{2 \pi}} \int_{-\infty}^{\infty} e^{-\beta\left(x+\frac{i s \ln \alpha}{2 \beta}\right)^{2}-\frac{s^{2}(\ln \alpha)^{2}}{4 \beta}} d x \\
& =\frac{N e^{-\frac{s^{2}(\ln \alpha)^{2}}{4 \beta}}}{\sqrt{2 \pi}} \int_{-\infty}^{\infty} e^{-\beta\left(x+\frac{i s \ln \alpha}{2 \beta}\right)^{2}} d x \\
& =\frac{N e^{-\frac{s^{2}(\ln \alpha)^{2}}{4 \beta}}}{\sqrt{2 \pi \beta}} \int_{-\infty}^{\infty} e^{-\mu^{2}} d \mu, \quad \text { where } \mu=\left(x \sqrt{\beta}+\frac{i s \ln \alpha}{2 \sqrt{\beta}}\right) \\
& =\frac{N e^{-\frac{s^{2}(\ln \alpha)^{2}}{4 \beta}}}{\sqrt{2 \pi \beta}} \sqrt{\pi}=\frac{N e^{-\frac{s^{2}(\ln \alpha)^{2}}{4 \beta}}}{\sqrt{2 \beta}}
\end{aligned}
$$




\subsection{Example}

Consider the modified Fourier transform of Box Function

$$
\begin{gathered}
f(x, \alpha)=\left\{\begin{array}{ll}
1 & |x| \leq r \\
0 & |x|>r
\end{array}\right\} \quad \text { Where } r>0 \\
\mathcal{F}_{\alpha}\{f(x, \alpha)\}=F(s, \alpha)=\frac{1}{\sqrt{2 \pi}} \int_{-\infty}^{\infty} f(x) \alpha^{-i s x} d x=\frac{1}{\sqrt{2 \pi}} \int_{-r}^{r} \alpha^{-i s x} d x \\
=\frac{\sqrt{2 \pi}}{\sqrt{2}}\left[\frac{\alpha^{-i s x} \ln \alpha}{-r}=\frac{-2}{\sqrt{2 \pi}(s \ln \alpha)}\left(\frac{-\alpha^{-i s r}+\alpha^{+i s r}}{2 i}\right)=\frac{-\sqrt{2} \sin r s}{\sqrt{\pi} s \ln \alpha}\right.
\end{gathered}
$$

\section{PROPERTIES OF MODIFIED FOURIER TRANSFORM}

\subsection{Linearity Property of Modified Fourier transform}

If $f_{1}(x, \alpha)$ and $f_{2}(x, \alpha)$ are two modified Fourier transformable functions, then for any two constants $c_{1}$ and $c_{2}$, we have

$\mathcal{F}_{\alpha}\left\{c f(x, \alpha)+c_{2} f_{2}(x, \alpha)\right\}=c \mathcal{F}_{\alpha}\{f(x, \alpha)\}+c_{2} \mathcal{F}_{\alpha}\left\{f_{2}(x, \alpha)\right\}$

\section{Proof.}

We can simply write

$$
\begin{aligned}
& \mathcal{F}_{\alpha}\left\{c f(x, \alpha)+c_{2} f_{2}(x, \alpha)\right\}=\frac{1}{\sqrt{2 \pi}} \int_{-\infty}^{\infty}\left(c f(x, \alpha)+c_{2} f_{2}(x, \alpha)\right) \alpha^{-i s x} d \\
& =c \frac{1}{\sqrt{2 \pi}} \int_{-\infty}^{\infty} f(x, \alpha) \alpha^{-i s x} d x+c_{2} \frac{1}{\sqrt{2 \pi}} \int_{-\infty}^{\infty} f_{2}(x, \alpha) \alpha^{-i s x} d x \\
& =c \mathcal{F}_{\alpha}\{f(x, \alpha)\}+c_{2} \mathcal{F}_{\alpha}\left\{f_{2}(x, \alpha)\right\}
\end{aligned}
$$

\subsection{Symmetry Modified Fourier transform}

$$
\mathrm{F}(x, \alpha) \stackrel{\mathcal{F}_{\alpha}}{\Leftrightarrow} f(-8, \alpha)
$$

Proof.

$$
\begin{aligned}
& f(x, \alpha)=\frac{}{\sqrt{2 \pi}} \int_{-\infty}^{\infty} \mathrm{F}(s, \alpha) \alpha^{i s x} d s \Rightarrow f(s, \alpha)=\frac{}{\sqrt{2 \pi}} \int_{-\infty}^{\infty} \mathrm{F}(x, \alpha) \alpha^{i s x} d x \\
& \Rightarrow f(-s, \alpha)=\frac{}{\sqrt{2 \pi}} \int_{-\infty}^{\infty} \mathrm{F}(x, \alpha) \alpha^{-i s x} d x=\mathcal{F}_{\alpha}\{\mathrm{F}(x, \alpha)\}
\end{aligned}
$$




\subsection{Conjugation property of Modified Fourier transform}

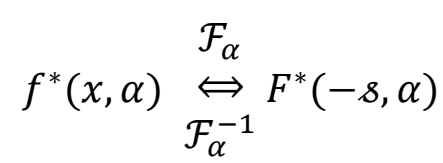

Proof.

$f^{*}(x, \alpha)=\frac{\overline{\sqrt{2 \pi}}}{\sqrt{2 \infty}} \int_{-\infty}^{\infty} F^{*}(s, \alpha) \alpha^{-i s x} d s=\frac{\pi}{\sqrt{2 \pi}} \int_{-\infty}^{\infty} F^{*}(-s, \alpha) \alpha^{i s x} d s$

$=\mathcal{F}_{\alpha}^{-1}\left\{f^{*}(x, \alpha)\right\}=F^{*}(-s, \alpha)$

\subsection{Scaling Property Modified Fourier transform}

$f(k x, \alpha) \underset{\mathcal{F}_{\alpha}^{-1}}{\stackrel{\mathcal{F}_{\alpha}}{\Leftrightarrow}} \overline{|k|} \mathrm{F}\left(\frac{s}{k}, \alpha\right)$

Proof.

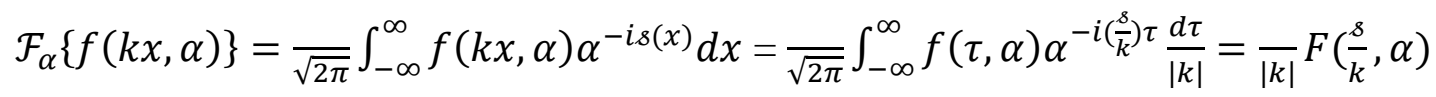

\subsection{Corollary}

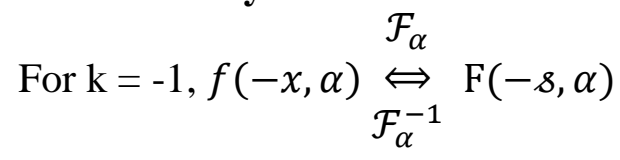

\subsection{Derivatives of Modified Fourier transform}

(i) $(-i x \ln \alpha)^{n} f(x, \alpha) \underset{\mathcal{F}_{\alpha}^{-1}}{\stackrel{\mathcal{F}_{\alpha}}{\Leftrightarrow}} \frac{\mathrm{d}^{n}}{\mathrm{~d} s^{\mathrm{n}}} \mathrm{F}(s, \alpha) \quad$ (ii) $\quad \frac{\mathrm{d}^{n}}{\mathrm{~d} x^{\mathrm{n}}} f(x, \alpha) \underset{\mathcal{F}_{\alpha}^{-1}}{\stackrel{\mathcal{F}_{\alpha}}{\Leftrightarrow}}(i \sin \alpha)^{n} \mathrm{~F}(s, \alpha)$

Proof (i).

$\mathrm{F}(s, \alpha)=\mathcal{F}_{\alpha}(f(x, \alpha))=\frac{1}{\sqrt{2 \pi}} \int_{-\infty}^{\infty} f(x, \alpha) \alpha^{-i s x} d x \rightarrow \frac{\mathrm{d}^{n}}{\mathrm{~d} s^{\mathrm{n}}} \mathrm{F}(s, \alpha)$

$=\frac{1}{\sqrt{2 \pi}} \int_{-\infty}^{\infty} f(x, \alpha)(-i x \ln \alpha)^{n} \alpha^{-i s x} d x$

$=\mathcal{F}_{\alpha}\left((-i x \ln \alpha)^{n} f(x, \alpha)\right)$

Proof (ii).

$f(x, \alpha)=\mathcal{F}_{\alpha}^{-1}(\mathrm{~F}(s, \alpha))=\frac{}{\sqrt{2 \pi}} \int_{-\infty}^{\infty} \mathrm{F}(s, \alpha) \alpha^{i s x} d s \rightarrow \frac{\mathrm{d}^{n}}{\mathrm{~d} x^{\mathrm{n}}} f(x, \alpha)$ 


$$
\begin{aligned}
& =\frac{\pi}{\sqrt{2 \pi}} \int_{-\infty}^{\infty} \mathrm{F}(s, \alpha)(i s \ln \alpha)^{n} \alpha^{i s x} d s \\
& =\mathcal{F}_{\alpha}^{-1}\left((i \operatorname{sln} \alpha)^{n} \mathrm{~F}(s, \alpha)\right)
\end{aligned}
$$

4.7 Definition (Convolution property of modified Fourier transform) The convolution of $f_{1}(x, \alpha)$ and $f_{2}(x, \alpha)$ where $f_{1}(x, \alpha)$ and $f_{2}(x, \alpha)$ are the Fourier transformable functions is represented by $f_{1}(x, \alpha) * f_{2}(x, \alpha)$ and is defined as

$$
\left(f * f_{2}\right)((x, \alpha))=\frac{1}{\sqrt{2 \pi}} \int_{-\infty}^{\infty} f(\tau, \alpha) f_{2}(x-\tau, \alpha) d \tau
$$

\subsection{Convolution Theorem for Modified Fourier transform}

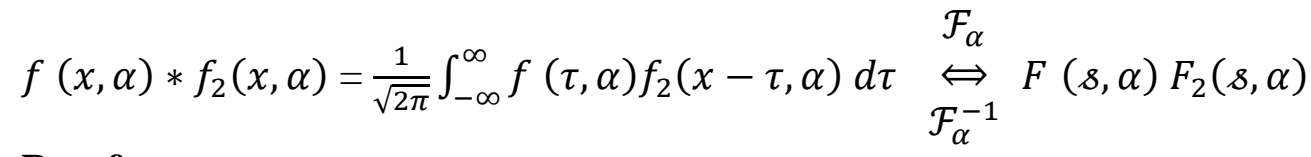

\section{Proof.}

$$
\begin{aligned}
& \mathcal{F}_{\alpha}\left(f_{1}(x, \alpha) * f_{2}(x, \alpha)\right)=\mathcal{F}_{\alpha}\left(\frac{1}{\sqrt{2 \pi}} \int_{-\infty}^{\infty} f(\tau, \alpha) f_{2}(x-\tau, \alpha) d \tau\right) \\
& =\frac{-}{2 \pi} \int_{-\infty}^{\infty} \alpha^{-i s x}\left(\int_{-\infty}^{\infty} f(\tau, \alpha) f_{2}(x-\tau, \alpha) d \tau\right) d x \\
& =\frac{-}{2 \pi} \int_{-\infty}^{\infty} f(\tau, \alpha) \alpha^{-i s \tau} \int_{-\infty}^{\infty} f_{2}(x-\tau, \alpha) \alpha^{-i s(x-\tau)} d x d \tau \\
& =\frac{-}{2 \pi} \int_{-\infty}^{\infty} f(\tau, \alpha) \alpha^{-i s \tau}\left(\int_{-\infty}^{\infty} f_{2}(u, \alpha) \alpha^{-i s(u)} d u\right) d \tau \\
& =\left(\frac{\pi}{\sqrt{2 \pi}} \int_{-\infty}^{\infty} f(\tau, \alpha) \alpha^{-i s \tau} d \tau\right) F_{2}(s, \alpha)=F_{1}(s, \alpha) F_{2}(s, \alpha)
\end{aligned}
$$

\subsection{Perseval,s Formulae for Modified Fourier transform}

(i) $\quad \int_{-\infty}^{\infty} f(x, \alpha) f_{2}{ }^{*}(x, \alpha) d x=\int_{-\infty}^{\infty} F(s, \alpha) F_{2}{ }^{*}(s, \alpha) d s$

(ii) $\int_{-\infty}^{\infty} f(x, \alpha) f_{2}(x, \alpha) d x=\int_{-\infty}^{\infty} F(s, \alpha) F_{2}(-s, \alpha) d s=\int_{-\infty}^{\infty} F(-s, \alpha) F_{2}(s, \alpha) d s$

\section{Proof (i).}

$$
\begin{aligned}
& f_{2}{ }^{*}(x, \alpha)=\frac{1}{\sqrt{2 \pi}} \int_{-\infty}^{\infty} F_{2}{ }^{*}(s, \alpha) \alpha^{-i s x} d s, \quad F(s, \alpha)=\frac{1}{\sqrt{2 \pi}} \int_{-\infty}^{\infty} f(x, \alpha) \alpha^{-i s x} d x \\
& \int_{-\infty}^{\infty} f(x, \alpha) f_{2}{ }^{*}(x, \alpha) d x=\frac{\pi}{\sqrt{2 \pi}} \int_{-\infty}^{\infty} f(x, \alpha) \int_{-\infty}^{\infty} F_{2}{ }^{*}(s, \alpha) \alpha^{-i s x} d s d x \\
& =\frac{\overline{\sqrt{2 \pi}}}{\sqrt{2 \pi}} \int_{-\infty}^{\infty} F_{2}^{*}(s, \alpha) \int_{-\infty}^{\infty} f(x, \alpha) \alpha^{-i s x} d x d s
\end{aligned}
$$


$=\int_{-\infty}^{\infty} F(s, \alpha) F_{2}^{*}(s, \alpha) d s$

Proof (ii).

$F(s, \alpha)=\frac{1}{\sqrt{2 \pi}} \int_{-\infty}^{\infty} f(x, \alpha) \alpha^{-i s x} d x \rightarrow F(-s, \alpha)=\frac{1}{\sqrt{2 \pi}} \int_{-\infty}^{\infty} f(x, \alpha) \alpha^{i s x} d x$

$\int_{-\infty}^{\infty} f(x, \alpha) f_{2}(x, \alpha) d x=\frac{\overline{\sqrt{2 \pi}}}{\sqrt{2 \infty}} \int_{-\infty}^{\infty} f(x, \alpha) \int_{-\infty}^{\infty} F_{2}(s, \alpha) \alpha^{i s x} d s d x$

$=\frac{\overline{\sqrt{2 \pi}}}{\sqrt{2 \pi}} \int_{-\infty}^{\infty} F_{2}(s, \alpha) \int_{-\infty}^{\infty} f(x, \alpha) \alpha^{i s x} d x d s$

$=\int_{-\infty}^{\infty} F(-s, \alpha) F_{2}(s, \alpha) d s$

\subsection{Shifting Property of Modified Fourier transform}

For any $k$ real

(i) $f(x-k, \alpha) \stackrel{\mathcal{F}_{\alpha}}{\Leftrightarrow} \alpha^{-i s k} \mathrm{~F}(s, \alpha) \quad$ (ii) $f(x, \alpha) \alpha^{i k x} \underset{\mathcal{F}_{\alpha}^{-1}}{\stackrel{\mathcal{F}_{\alpha}}{\Leftrightarrow}} \mathrm{F}(s-k, \alpha)$

Proof (i).

$\mathcal{F}_{\alpha}\{f(x-k, \alpha)\}=\frac{1}{\sqrt{2 \pi}} \int_{-\infty}^{\infty} f(x-k, \alpha) \alpha^{-i s x} d x$

Put $\tau=x-k, d \tau=d x$

$=\frac{1}{\sqrt{2 \pi}} \int_{-\infty}^{\infty} f(\tau, \alpha) \alpha^{-i s(\tau+k)} d \tau=\alpha^{-i s k} \frac{1}{\sqrt{2 \pi}} \int_{-\infty}^{\infty} f(\tau, \alpha) \alpha^{-i s(\tau)} d \tau=\alpha^{-i s k} \mathrm{~F}(s, \alpha)$

Proof (ii).

$\mathcal{F}_{\alpha}\left\{f(x, \alpha) \alpha^{i k x}\right\}=\frac{1}{\sqrt{2 \pi}} \int_{-\infty}^{\infty} f(x, \alpha) \alpha^{i k x} \alpha^{-i s x} d x$

$=\frac{1}{\sqrt{2 \pi}} \int_{-\infty}^{\infty} f(x, \alpha) \alpha^{-i(s-k)} d x$

$=\mathrm{F}(s-k, \alpha)$.

\subsection{Modulation Property of Modified Fourier transform}

$f(x, \alpha) \cos \left(s_{o} x \ln \alpha\right) \underset{\mathcal{F}_{\alpha}^{-1}}{\stackrel{\mathcal{F}_{\alpha}}{\Leftrightarrow}} \frac{1}{2}\left[\mathrm{~F}\left(s+s_{o} x \ln \alpha, \alpha\right)+\mathrm{F}\left(s-s_{o} x \ln \alpha, \alpha\right)\right]$

\section{Proof.}


$\mathcal{F}_{\alpha}\left\{f(x, \alpha) \cos \left(s_{o} x \ln \alpha\right)\right\}=\mathcal{F}_{\alpha}\left\{f(x, \alpha) \frac{1}{2}\left[e^{i s_{o} x \ln \alpha}+e^{-i s_{o} x \ln \alpha}\right]\right\}$

$=\frac{-}{2}\left[\mathrm{~F}\left(s+s_{o} x \ln \alpha, \alpha\right)+\mathrm{F}\left(s-s_{o} x \ln \alpha, \alpha\right)\right]$, using shifting property with $\boldsymbol{k}= \pm \boldsymbol{s}_{\boldsymbol{o}}$

\subsection{If $\boldsymbol{f}(\boldsymbol{x}, \alpha)$ is even then $\boldsymbol{F}(\boldsymbol{s}, \alpha)$ in real}

\section{Proof.}

$$
\begin{aligned}
& F(s, \alpha)=\mathcal{F}_{\alpha}\{f(x, \alpha)\}=\frac{\pi}{\sqrt{2 \pi}} \int_{-\infty}^{\infty} f(x, \alpha) \alpha^{-i s(x)} d x \\
& =\frac{1}{\sqrt{2 \pi}}\left\{\int_{-\infty}^{0} f(x, \alpha) \alpha^{-i s(x)} d x+\int_{0}^{\infty} f(x, \alpha) \alpha^{-i s(x)} d x\right\}
\end{aligned}
$$

Put $x=-x$ in the first integral then as $x \rightarrow 0$ then $-x \rightarrow 0$ and as $x \rightarrow-\infty$ then $-x \rightarrow \infty$

$$
\begin{aligned}
& =\frac{}{\sqrt{2 \pi}}\left\{-\int_{\infty}^{0} f(-x, \alpha) \alpha^{+i s(x)} d x+\int_{0}^{\infty} f(x, \alpha) \alpha^{-i s(x)} d x\right\} \\
& =\frac{}{\sqrt{2 \pi}} \int_{0}^{\infty} f(x, \alpha)\left(\alpha^{i s(x)}+\alpha^{-i s(x)}\right) d x \quad \because\left\{\begin{array}{c}
\boldsymbol{f}(\boldsymbol{x}, \alpha)=\boldsymbol{f}(-\boldsymbol{x}, \alpha) \\
\int_{\infty}^{\mathbf{0}} \boldsymbol{f}=-\int_{\mathbf{0}}^{\infty} \boldsymbol{f}
\end{array}\right\} \\
& =\frac{2}{\sqrt{2 \pi}} \int_{0}^{\infty} f(x, \alpha)\left(\frac{\alpha^{i s(x)}+\alpha^{-i s(x)}}{2}\right) d x=\frac{2}{\sqrt{2 \pi}} \int_{0}^{\infty} f(x, \alpha)\left(\frac{e^{i s(x) \alpha}+e^{-i s(x) \ln \alpha}}{2}\right) d x \\
& =\sqrt{\frac{2}{\pi}}\left\{\int_{0}^{\infty} f(x, \alpha) \cos (s x \ln \alpha) d x\right\}
\end{aligned}
$$

\subsection{If $\boldsymbol{f}(\boldsymbol{x}, \alpha)$ is odd, then $\boldsymbol{F}(\boldsymbol{s}, \alpha)$ is pure imaginary}

\section{Proof.}

$$
\begin{array}{r}
F(s, \alpha)=\mathcal{F}_{\alpha}\{f(x, \alpha)\}=\frac{1}{\sqrt{2 \pi}} \int_{-\infty}^{\infty} f(x, \alpha) \alpha^{-i s(x)} d x \\
=\frac{1}{\sqrt{2 \pi}}\left\{\int_{-\infty}^{0} f(x, \alpha) \alpha^{+i s(x)} d x+\int_{0}^{\infty} f(x, \alpha) \alpha^{-i s(x)} d x\right\}
\end{array}
$$

Put $x=-x$ in the first integral then $d x=-d x$ and as $x \rightarrow 0$ we have $-x \rightarrow 0$ and as $x \rightarrow-\infty$ then $-x \rightarrow \infty$ 


$$
\begin{aligned}
& F(s, \alpha)=\frac{1}{\sqrt{2 \pi}}\left\{-\int_{\infty}^{0} f(-x, \alpha) \alpha^{+i s(x)} d x+\int_{0}^{\infty} f(x, \alpha) \alpha^{-i s(x)} d x\right\} \\
& =\frac{1}{\sqrt{2 \pi}} \int_{0}^{\infty} f(x, \alpha)\left(-\alpha^{i s(x)}+\alpha^{-i s(x)}\right) d x=-\frac{2 i}{\sqrt{2 \pi}} \int_{0}^{\infty} f(x, \alpha)\left(\frac{\alpha^{i s(x)}-\alpha^{-i s(x)}}{2 i}\right) d x \\
& =-i \sqrt{\frac{2}{\pi}} \int_{0}^{\infty} f(x, \alpha)\left(\frac{\alpha^{i s(x)}-\alpha^{-i s(x)}}{2 i}\right) d x=-i \sqrt{\frac{2}{\pi}}\left\{\int_{0}^{\infty} f(x, \alpha)\left(\frac{e^{i s x \ln \alpha}-e^{-i s x \ln \alpha}}{2 i}\right) d x\right\} \\
& =-i \sqrt{\frac{2}{\pi}}\left\{\int_{0}^{\infty} f(x, \alpha) \sin (s x \ln \alpha) d x\right\}
\end{aligned}
$$

\section{DERIVATION OF MODIFIED FOURIER INTEGRAL THEOREM}

Consider the inverse modified Fourier Transform

$$
\begin{aligned}
& f(x, \alpha)=\frac{1}{\sqrt{2 \pi}} \int_{-\infty}^{\infty} \mathrm{F}(s, \alpha) \alpha^{i s x} d s \\
& =\frac{1}{\sqrt{2 \pi}}\left\{\int_{-\infty}^{0} \mathrm{~F}(s, \alpha) \alpha^{i s x} d s+\int_{0}^{\infty} \mathrm{F}(s, \alpha) \alpha^{i s x} d s\right\}
\end{aligned}
$$

Replacing ' $s$ ' by ' $-s$ ' in the first integral of equation (1) then we have

$$
\begin{aligned}
& f(x, \alpha)=\frac{1}{\sqrt{2 \pi}}\left\{\int_{-\infty}^{0} F_{2}{ }^{*}(s, \alpha) \alpha^{-i s x} d s+\int_{0}^{\infty} \mathrm{F}(s, \alpha) \alpha^{i s x} d s\right\} \\
& =\frac{1}{\sqrt{2 \pi}}\left\{\int_{0}^{\infty} F_{2}{ }^{*}(s, \alpha) \alpha^{-i s x} d s+\int_{0}^{\infty} \mathrm{F}(s, \alpha) \alpha^{i s x} d s\right\} \\
& \mathrm{F}(s, \alpha) \alpha^{i s x}=\frac{1}{\sqrt{2 \pi}} \int_{-\infty}^{\infty} f(r, \alpha) \alpha^{-i s(r-x)} d r \\
& F_{2}{ }^{*}(s, \alpha) \alpha^{-i s x}=\frac{1}{\sqrt{2 \pi}} \int_{-\infty}^{\infty} f(r, \alpha) \alpha^{i s(r-x)} d r
\end{aligned}
$$

Substituting the values of equations (3) and (4) into equation (2)

$$
\begin{aligned}
& f(x, \alpha)=\frac{1}{2 \pi} \int_{0}^{\infty}\left\{\int_{-\infty}^{\infty} f(r, \alpha) \alpha^{-i s(r-x)} d r+\int_{-\infty}^{\infty} f(r, \alpha) \alpha^{i s(r-x)} d r\right\} d s \\
& =\frac{1}{\pi} \int_{0}^{\infty} \int_{-\infty}^{\infty} f(r, \alpha)\left\{\frac{\alpha^{i s(r-x)}+\alpha^{-i s(r-x)}}{2}\right\} d r d s \\
& =\frac{1}{\pi} \int_{0}^{\infty} \int_{-\infty}^{\infty} f(r, \alpha)\left\{\frac{e^{i s(r-x) \ln \alpha}+e^{-i s(r-x) \ln \alpha}}{2}\right\} d r d s
\end{aligned}
$$




$$
\left.=\frac{1}{\pi} \int_{0}^{\infty} \int_{-\infty}^{\infty} f(r, \alpha) \cos (s(r-x) \ln \alpha)\right\} d r d s
$$

Equation (5) is the modified Fourier integral identity.

\subsection{Derivation of Modified Fourier Cosine Integral}

Let $f(x, \alpha)$ is an even function then

$$
\begin{aligned}
f(x, \alpha) & \left.=\frac{1}{\pi} \int_{0}^{\infty} \int_{-\infty}^{\infty} f(r, \alpha) \cos (s(r-x) \ln \alpha)\right\} d r d s \\
& =\frac{1}{\pi} \int_{0}^{\infty} \int_{-\infty}^{\infty} f(r, \alpha) \cos (s r \ln \alpha) \cos (s x \ln \alpha) d r d s \\
& =\frac{1}{\pi} \int_{0}^{\infty} \cos (s x \ln \alpha) \int_{-\infty}^{\infty} f(r, \alpha) \cos (s r \ln \alpha) d r d s \\
& =\frac{2}{\pi} \int_{0}^{\infty} \cos (s x \ln \alpha) \int_{0}^{\infty} f(r, \alpha) \cos (s r \ln \alpha) d r d s
\end{aligned}
$$

Equation (6) is the modified Fourier Cosine integral identity.

\subsection{Derivation of Modified Fourier Sine Integral}

Let $f(x, \alpha)$ is an odd function then

$$
\begin{aligned}
f(x, \alpha) & \left.=\frac{1}{\pi} \int_{0}^{\infty} \int_{-\infty}^{\infty} f(r, \alpha) \cos (s(r-x) \ln \alpha)\right\} d r d s \\
& =\frac{1}{\pi} \int_{0}^{\infty} \int_{-\infty}^{\infty} f(r, \alpha) \sin (s r \ln \alpha) \sin (s x \ln \alpha) d r d s \\
& =\frac{1}{\pi} \int_{0}^{\infty} \sin (s x \ln \alpha) \int_{-\infty}^{\infty} f(r, \alpha) \sin (s r \ln \alpha) d r d s \\
& =\frac{2}{\pi} \int_{0}^{\infty} \sin (s x \ln \alpha) \int_{0}^{\infty} f(r, \alpha) \sin (s r \ln \alpha) d r d s
\end{aligned}
$$

Equation (7) is the modified Fourier sine integral identity.

\subsection{Derivation of Modified Fourier Cosine Transform and its inverse}

We can obtain the modified Fourier cosine transform from modified Fourier cosine integral formula

$f(x, \alpha)=\frac{2}{\pi} \int_{0}^{\infty} \cos (s x \ln \alpha) \int_{0}^{\infty} f(r, \alpha) \cos (s r \ln \alpha) d r d s$ 


$$
=\sqrt{\frac{2}{\pi}} \int_{0}^{\infty} \cos (s x \ln \alpha) \cdot \sqrt{\frac{2}{\pi}} \int_{0}^{\infty} f(r, \alpha) \cos (s r \ln \alpha) d r d s
$$

The second integral of equation (8) is defined as Fourier Cosine Transform which is denoted by $F_{c}(s, \alpha)$ and given by

$$
F_{c}(8, \alpha)=\sqrt{\frac{2}{\pi}} \int_{0}^{\infty} f(x, \alpha) \cos (s x \ln \alpha) \mathrm{d} x
$$

While inverse modified Fourier cosine transform is obtained by substituting $F_{c}(8, \alpha)$ in equation (8)

$$
f_{c}(x, \alpha)=\sqrt{\frac{2}{\pi}} \int_{0}^{\infty} F_{c}(s, \alpha) \cos (s x \ln \alpha) d s
$$

\subsection{Derivation of Modified Fourier Sine Transform and its inverse}

We can obtain the modified Fourier sine transform from modified Fourier sine integral formula

$$
\begin{aligned}
f(x, \alpha) & =\frac{2}{\pi} \int_{0}^{\infty} \sin (s x \ln \alpha) \int_{0}^{\infty} f(r, \alpha) \sin (s r \ln \alpha) d r d s \\
& =\sqrt{\frac{2}{\pi}} \int_{0}^{\infty} \sin (s x \ln \alpha) \cdot \sqrt{\frac{2}{\pi}} \int_{0}^{\infty} f(r, \alpha) \sin (s r \ln \alpha) d r d s
\end{aligned}
$$

The second integral of equation (9) is defined as Fourier cosine transform which is denoted by $F_{S}(8, \alpha)$ and given by

$$
F_{S}(s, \alpha)=\sqrt{\frac{2}{\pi}} \int_{0}^{\infty} f(x, \alpha) \sin (s x \ln \alpha) \mathrm{d} x
$$

While inverse modified Fourier cosine transform is obtained by substituting $F_{S}(s, \alpha)$ in equation (9).

$$
f_{S}(x, \alpha)=\sqrt{\frac{2}{\pi}} \int_{0}^{\infty} F_{S}(s, \alpha) \sin (s x \ln \alpha) d s
$$

\section{ALGEBRA OF MODIFIED FOURIER TRANSFORM}

\subsection{Theorem}

Prove that the set which contains the whole modified Fourier transformable functions under the convolution operation ' $*$ ' is a commutative semi group.

\section{Proof.}

Let $\mathrm{T}$ is the set that contains the whole modified Fourier transformable functions

\section{Commutative Property:}


commutativity holds in $\mathrm{T}$ under the convolution operation ' $*$ ' i.e.

$\left(f_{1} * f_{2}\right)((x, \alpha))=\frac{1}{\sqrt{2 \pi}} \int_{-\infty}^{\infty} f_{1}(\tau, \alpha) f_{2}(x-\tau, \alpha) d \tau$

Let $u=x-\tau$, So $\mathrm{d} u=-d \tau$.

$\left(f_{1} * f_{2}\right)(x, \alpha)=\frac{1}{\sqrt{2 \pi}} \int_{\infty}^{-\infty} f_{1}(x-u, \alpha) f_{2}(u, \alpha)(-d u)$

$=\frac{1}{\sqrt{2 \pi}} \int_{-\infty}^{\infty} f_{2}(u, \alpha) f_{1}(x-u, \alpha) d u=\left(f_{2} * f_{1}\right)((x, \alpha))$

\section{Associative Property:}

associativity holds in T under the convolution operation ' $*$ ' i.e.

$$
\begin{aligned}
\left(\left(f_{1} * f_{2}\right) * f_{3}\right)(x, \alpha) & =\frac{1}{\sqrt{2 \pi}} \int_{-\infty}^{\infty}\left(f_{1} * f_{2}\right)(\tau, \alpha) f_{3}(x-\tau, \alpha) d \tau \\
& =\frac{1}{\sqrt{2 \pi}} \int_{-\infty}^{\infty}\left(\frac{1}{\sqrt{2 \pi}} \int_{-\infty}^{\infty} f_{1}(u, \alpha) f_{2}(\tau-u, \alpha) d u\right) f_{3}(x-\tau, \alpha) d \tau \\
& =\frac{1}{2 \pi} \int_{-\infty}^{\infty} \int_{-\infty}^{\infty} f_{1}(u, \alpha) f_{2}(\tau-u, \alpha) f_{3}(x-\tau, \alpha) d u d \tau \\
& =\frac{1}{2 \pi} \int_{-\infty}^{\infty} \int_{-\infty}^{\infty} f_{1}(u, \alpha) f_{2}(\tau-u, \alpha) f_{3}(x-\tau, \alpha) d \tau d u \\
& =\frac{1}{2 \pi} \int_{-\infty}^{\infty} f_{1}(u, \alpha)\left(\int_{-\infty}^{\infty} f_{2}(\tau, \alpha) f_{3}(x-u-\tau, \alpha) d \tau\right) d u \\
& =\frac{1}{2 \pi} \int_{-\infty}^{\infty} f_{1}(u, \alpha)\left(f_{2} * f_{3}\right)(x-u, \alpha) d u \\
& =\left(f_{1} *\left(f_{2} * f_{3}\right)\right)(x, \alpha)
\end{aligned}
$$

\section{Distributive Property:}

distrbutivity holds in T under the convolution operation ' $*$ ' i.e.

$$
\begin{aligned}
& \left(f_{1} *\left(f_{2}+f_{3}\right)\right)(x, \alpha)==\frac{1}{\sqrt{2 \pi}} \int_{-\infty}^{\infty} f_{1}(\tau, \alpha)\left(f_{2}+f_{3}\right)(x-\tau, \alpha) d \tau \\
& =\frac{1}{\sqrt{2 \pi}} \int_{-\infty}^{\infty}\left(f_{1}(\tau, \alpha) f_{2}(x-\tau, \alpha) d \tau+\frac{1}{\sqrt{2 \pi}} \int_{-\infty}^{\infty}\left(f_{1}(\tau, \alpha) f_{3}(x-\tau, \alpha) d \tau\right.\right. \\
& =\left(f_{1} * f_{2}\right)(x, \alpha)+\left(f_{1} * f_{3}\right)(x, \alpha)
\end{aligned}
$$

\section{Identity Property:}


identity property holds in $\mathrm{T}$ under the convolution operation ' $*$ ' i.e.

$(f * \delta)(x, \alpha)=\frac{1}{\sqrt{2 \pi}} \int_{-\infty}^{\infty} f(\tau, \alpha) \delta(x-\tau, \alpha) d \tau=(\delta * f)(x, \alpha)=f(x, \alpha)$

Where $\delta$ is Kronecker delta.

\section{Theorem 6.2}

Prove that the set which contains the whole modified Fourier transformable functions under the operation ' + 'is an Abelian group.

\section{Proof.}

Let $\mathrm{T}$ is the set that contains the whole modified Fourier transformable functions.

\section{Closure property:}

Let $f_{1}(x, \alpha), f_{2}(x, \alpha) \in \mathrm{T}$, then there exists $s_{1}$ and $s_{2}$ such that $F\left(s_{1}, \alpha\right)=\frac{1}{\sqrt{2 \pi}} \int_{-\infty}^{\infty} f_{1}(x, \alpha) \alpha^{-i s_{1}(x)} d x$, and $F\left(s_{2}, \alpha\right)=\frac{1}{\sqrt{2 \pi}} \int_{-\infty}^{\infty} f_{2}(x, \alpha) \alpha^{-i s_{2}(x)} d x$ exist.

Evidently, if we take $s=\max \left(s_{1}, s_{2}\right)$, then $F\left(s_{1}, \alpha\right)+F\left(s_{2}, \alpha\right)=\frac{1}{\sqrt{2 \pi}} \int_{-\infty}^{\infty}\left(f_{1}(x, \alpha)+f_{2}(x, \alpha)\right) \alpha^{-i s(x)} d x$ exists $\Rightarrow f_{1}(x, \alpha)+f_{2}(x, \alpha) \in \mathrm{T} \Rightarrow$ closure property holds in $\mathrm{T}$.

\section{Associative property:}

The associative property is evident, i.e. for $f_{1}(x, \alpha), f_{2}(x, \alpha), f_{3}(x, \alpha) \in \mathrm{T}$

$$
\begin{aligned}
& \left(F\left(s_{1}, \alpha\right)+F\left(s_{2}, \alpha\right)\right)+F\left(s_{3}, \alpha\right) \\
& =\left(\frac{1}{\sqrt{2 \pi}} \int_{-\infty}^{\infty} f_{1}(x, \alpha) \alpha^{-i s_{1}(x)} d x+\frac{1}{\sqrt{2 \pi}} \int_{-\infty}^{\infty} f_{2}(x, \alpha) \alpha^{-i s_{2}(x)} d x\right)+\frac{1}{\sqrt{2 \pi}} \int_{-\infty}^{\infty} f_{3}(x, \alpha) \alpha^{-i s_{3}(x)} d x \\
& =\frac{1}{\sqrt{2 \pi}} \int_{-\infty}^{\infty}\left(f_{1}(x, \alpha)+f_{2}(x, \alpha)\right) \alpha^{-i\left(s_{1}+s_{2}\right)(x)} d x+\frac{1}{\sqrt{2 \pi}} \int_{-\infty}^{\infty} f_{3}(x, \alpha) \alpha^{-i s_{3}(x)} d x \\
& =\frac{1}{\sqrt{2 \pi}} \int_{-\infty}^{\infty}\left(\left(f_{1}(x, \alpha)+f_{2}(x, \alpha)\right)+f_{3}(x, \alpha)\right) \alpha^{-i\left(\left(\left(s_{1}+s_{2}\right)\right)+s_{3}\right)(x)} d x \\
& =\frac{1}{\sqrt{2 \pi}} \int_{-\infty}^{\infty}\left(f_{1}(x, \alpha)+\left(f_{2}(x, \alpha)+f_{3}(x, \alpha)\right) \alpha^{-i\left(s_{1}+\left(s_{2}+s_{3}\right)\right)(x)} d x\right.
\end{aligned}
$$




$$
\begin{aligned}
& =\frac{\sqrt{2 \pi}}{\sqrt{2 \pi}} \int_{-\infty}^{\infty} f(x, \alpha) \alpha^{-i s_{1}(x)} d x+\left(\frac{1}{\sqrt{2 \pi}} \int_{-\infty}^{\infty}\left(f_{2}(x, \alpha)+f_{3}(x, \alpha)\right) \alpha^{-i\left(s_{2}+s_{3}\right)(x)} d x\right) \\
& =\frac{1}{\sqrt{2 \pi}} \int_{-\infty}^{\infty} f(x, \alpha) \alpha^{-i s_{1}(x)} d x+\left(\frac{}{\sqrt{2 \pi}} \int_{-\infty}^{\infty} f_{2}(x, \alpha) \alpha^{-i s_{2}(x)} d x+\frac{}{\sqrt{2 \pi}} \int_{-\infty}^{\infty} f_{3}(x, \alpha) \alpha^{-i s_{3}(x)} d x\right) \\
& \left.=F(s, \alpha)+\left(F\left(s_{2}, \alpha\right)\right)+F\left(s_{3}, \alpha\right)\right)
\end{aligned}
$$

\section{Identity property:}

Identity or null element is the ordinary ' 0 ', i.e. for $f(x, \alpha) \in \mathrm{T}$ we have

$$
\overline{\sqrt{2 \pi}} \int_{-\infty}^{\infty} f(x, \alpha) \alpha^{-i s(x)} d x+0=0+\frac{1}{\sqrt{2 \pi}} \int_{-\infty}^{\infty} f(x, \alpha) \alpha^{-i s(x)} d x=F(s, \alpha)
$$

\section{Inverse Property:}

Let $f(x, \alpha) \in \mathrm{T}$ then there exists $-f(x, \alpha) \in \mathrm{T}$ such that

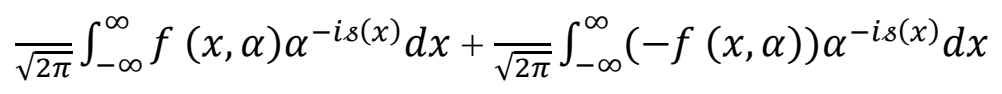

$=\frac{1}{\sqrt{2 \pi}} \int_{-\infty}^{\infty}(f(x, \alpha)-f(x, \alpha)) \alpha^{-i s(x)} d x=0$

$\Longrightarrow$ inverse property holds in $\mathrm{T}$.

\section{Commutative Property:}

$$
\begin{aligned}
& F(s, \alpha)+F\left(s_{2}, \alpha\right)=\frac{1}{\sqrt{2 \pi}} \int_{-\infty}^{\infty}\left(f_{1}(x, \alpha)+f_{2}(x, \alpha)\right) \alpha^{-i\left(s_{1}+s_{2}\right)(x)} d x \\
& =\frac{1}{\sqrt{2 \pi}} \int_{-\infty}^{\infty}\left(f_{2}(x, \alpha)+f(x, \alpha)\right) \alpha^{-i\left(s_{2}+s_{1}\right)(x)} d x=F\left(s_{2}, \alpha\right)+F(s, \alpha)
\end{aligned}
$$

$\Rightarrow$ Commutative property holds in T. Hence the theorem.

\section{CONCLUSION}

Fourier transform is a significant technique applied in huge areas of engineering and science. The Fourier transform has a wide range of applications, not only in applied mathematics, engineering and statistics but also in a variety of other fields, e.g., in the field of physics, a simple application of the modified Fourier transform might be to determine a beam's harmonic vibration. Since the Fourier transform is useful, there is a lot of literature on it, and many scholars have looked into its applications and properties. Following that, the aim of this paper is to demonstrate a modified Fourier transform which is more common and covers a broader range $(\alpha \in(0, \infty)-\{1\})$. Results gained by modified Fourier transform in the problems of applied mathematics and physics would be better précised and reliable. We will consider certain advanced problems concerning modified Fourier transform in upcoming 
papers. In future, we will describe a 2D modified Fourier transform and display its linearity property. Some new properties of the double Fourier transform can be studied further by interested authors.

The 2D modified Fourier transform of a function $f(\mathrm{x}, \mathrm{y}, \alpha)$ is defined a

$F(s, k, \alpha)=\int_{-\infty}^{\infty} \int_{-} f(x, y, \alpha) \alpha^{-i 2 \pi(s x+k y)} d x d y,(s, k>0)$

2D modified Fourier transform satisfies the linearity property

$\mathcal{F}_{\alpha}\left\{c f(x, y, \alpha)+c_{2} f_{2}(x, y, \alpha)\right\}=c \mathcal{F}_{\alpha}\{f(x, y, \alpha)\}+c_{2} \mathcal{F}_{\alpha}\left\{f_{2}(x, y, \alpha)\right\}$

where $c_{1}$ and $c_{2}$ are constants.

\section{REFERENCES}

[1] O. M. Bucci, \& M. D. Migliore. "A novel nonuniform fast fourier transform algorithm and its application to aperiodic arrays", IEEE Antennas and Wireless Propagation Letters, (16), 1472 - 1475 (2016).

[2] S. S.Ezhil, "Real Time Application of Fourier Transforms", Indonesian Journal of Electrical Engineering and Computer Science (IJEECS), (2), 574-577 (2017).

[3] M. E. Jakubauskas, D. R. Legates, \& J. H. Kastens, "Harmonic analysis of timeSeries AVHRR NDVI data", Photogrammetric engineering and remote sensing, (4), 461- 470 (2001).

[4] N. CHITTORA \& D. BABEL, “A Brief Study on Fourier Transform and Its Applications" (5), 1127-1131(2018).

[5] M. Guerquin-Kern, L. Lejeune, K. P. Pruessmann, \& M. Unser, "Realistic analytical Phantoms for parallel magnetic resonance imaging", IEEE Transactions on Medical Imaging, (3), 626-636 (2012).

[6] T. Benz, M. Kowarschik, J. Endres, , T. Redel, S. Demirci, \& N. Navab, “A Fourier-based approach to the angiographic assessment of flow diverter efficacy in the treatment of cerebral aneurysms", IEEE transactions on medical imaging, (9), 1788-1802 (2014).

[7] A. Gupta. "Fourier Transform and Its Application in Cell Phones", International Journal of Scientific and Research Publications, (1), 1-2 (2013).

[8] Vigouroux, Corinne, B. Langerock, C. Augusto, B. Aquino, Thomas Blumenstock, Zhibin Cheng, Martine De Mazière, Isabelle De Smedt et al. "TROPOMI-Sentinel-5 Precursor formaldehyde validation using an extensive network of ground-based Fouriertransform infrared stations." Atmospheric Measurement Techniques, (7), 3751-3767 (2020).

[9] T.Sakaemura, I. Mihara, \& T. Yamauchi. Microscopic Attenuated Total Reflection/Fourier Transform Infrared Imaging of Paper Containing a Polyacrylamide Dry Strength Resin. Sen'i Gakkaishi, (9), 252-255 (2009).

Received April 24, 2021 